\title{
Procesos de oxidación de Si mediante plasma de resonancia ciclotrónica de electrones
}

\author{
E. SAN ANDRÉS, A. DEL PRADO, A. J. BLÁZQUEZ, I. MÁRTIL, G. GONZÁLEZ-DÍAZ \\ Departamento de Física Aplicada III, Facultad de Ciencias Físicas, Universidad Complutense de Madrid, E-28040 Madrid, España.
}

\begin{abstract}
Se han fabricado estructuras MIS sobre Si (100) mediante un proceso en dos pasos: una primera exposición del sustrato de Si a un plasma ECR de oxígeno, que da lugar a la obtención de una capa de $\mathrm{SiO}_{\mathrm{x}}$ (en adelante $\mathrm{PO}_{\mathrm{SiO}}$ ), seguido de un depósito de nitruro de silicio ( $\mathrm{SiN} \mathrm{N}_{1.55^{\circ}}$ $\mathrm{H})$ mediante plasma ECR. La estructura MIS resultante es de la forma $\mathrm{Al} / \mathrm{SiN}_{1.55}: \mathrm{H} / \mathrm{PO}-\mathrm{SiO}_{x} / \mathrm{Si}$. Los dispositivos han sido caracterizados mediante la medida simultánea de las capacidades a alta y baja frecuencia, lo que permite conocer la calidad de la intercara $\mathrm{PO}-\mathrm{SiO} \times \mathrm{Si}$, calcular los espesores de la capa de $\mathrm{PO}-\mathrm{SiO}_{\mathrm{x}}$ y la velocidad de crecimiento del $\mathrm{SiN}_{\mathrm{x}}: \mathrm{H}$. Para caracterizar el proceso de oxidación se realizaron varias series de muestras variando en cada una un parámetro del proceso. Estos parámetros fueron: el tiempo de depósito del SiN $: \mathrm{H}$, el tiempo de oxidación, la temperatura del sustrato y el flujo total de $\mathrm{O}_{2}$

Asimismo, se ha estudiado la estructura de enlaces del dieléctrico apilado mediante espectroscopia infrarroja. El espectro del dieléctrico apilado mostró la superposición de dos picos: uno de menor intensidad asociado al PO-SiO con el máximo en $1056 \mathrm{~cm}^{-1}$, y otro debido al

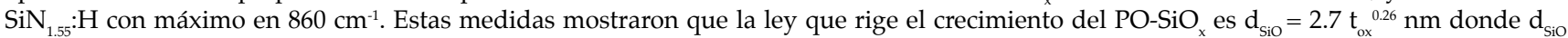
es el espesor de la capa de $\mathrm{PO}-\mathrm{SiO}_{\mathrm{x}}$ y $\mathrm{t}_{\mathrm{ox}}$ es el tiempo de oxidación en min. Por lo que respecta a las características eléctricas, las estructuras presentaron mínimos de la densidad de trampas en la intercara $\left(D_{\mathrm{it}}\right)$ cercanos a $10^{11} \mathrm{eV}^{-1} \mathrm{~cm}^{-2}$. Este valor es inferior al que presentaron las estructuras sin oxidar, del tipo $\mathrm{SiN}_{1.55}: \mathrm{H} / \mathrm{Si}$. Además, los dispositivos apilados mostraron un barrido del nivel de Fermi mayor y una histéresis prácticamente despreciable.
\end{abstract}

Palabras clave: Plasma ECR, oxidación por plasma, subóxido de Si, dieléctrico apilado.

\section{Si oxidation processes by electron cyclotron resonance plasmas}

MIS structures have been fabricated on $\mathrm{Si}$ (111) by a two-step process: first an exposition of the Si substrates to an ECR oxygen plasma was performed, which yields a layer of $\mathrm{SiO}_{\mathrm{x}}$ (in the following $\mathrm{PO}-\mathrm{SiO}_{\mathrm{x}}$ ); this process was followed by an ECR plasma silicon nitride deposition $\left(\mathrm{SiN}_{1.55}: \mathrm{H}\right)$. The resulting MIS structure is $\mathrm{Al} / \mathrm{SiN}_{1.55}: \mathrm{H} / \mathrm{PO}-\mathrm{SiO}_{\mathrm{x}} / \mathrm{Si}$. Devices have been characterized by the simultaneous measurement of the capacitance at high and low frequencies. This measurement lets us know the $\mathrm{PO}-\mathrm{SiO}_{\mathrm{x}} / \mathrm{Si}$ interface quality, calculate the thickness of the $\mathrm{PO}-\mathrm{SiO}_{x}$ layer and the growth rate of $\mathrm{SiN}_{1.5}: \mathrm{H}$. To characterize the oxidation process some series of samples were prepared. In each series a process parameter was varied. These parameters were: the $\mathrm{SiN}_{155}: \mathrm{H}$ deposition time, the ECR plasma oxidation duration, the substrate temperature and the total oxygen flux.

The bonding structure of the stacked dielectric has been studied by infrared spectroscopy. The stacked dielectrics spectra showed the superposition of two peaks: a less intense peak associated to the $\mathrm{PO}-\mathrm{SiO}_{x}$ layer with its maximum in $1056 \mathrm{~cm}^{-1}$, and another one due to the $\mathrm{SiN} \mathrm{N}_{1.5}: \mathrm{H}$ film with its maximum in $860 \mathrm{~cm}^{-1}$. These measurements showed that the PO-SiO ${ }_{x}$ growth law is $\mathrm{d}_{\mathrm{SiO}}=2.7 \mathrm{t}_{\mathrm{ox}}^{0.26} \mathrm{~nm}_{\text {where }} \mathrm{d}_{\mathrm{SiO}}$ is the PO-SiO layer thickness and $t_{o x}$ is the oxidation time in min. Concerning electrical characteristics, stacked MIS devices showed interface trap density minimums $\left(\mathrm{D}_{\mathrm{it}}\right)$ close to $10^{11} \mathrm{eV}^{-1} \mathrm{~cm}^{-2}$. This value is inferior to the one that non-oxydized devices $\left(\mathrm{Al} / \mathrm{SiN}{ }_{1.55}: \mathrm{H} / \mathrm{Si}\right)$ show. Also, stacked devices presented higher Fermi level sweeps and an electrical hysteresis almost insignificant.

Keywords:

\section{INTRODUCCIÓN}

La actual tendencia hacia la disminución del tamaño de los dispositivos MOSFET trae como consecuencia una disminución del espesor del $\mathrm{SiO}_{2}$ crecido térmicamente que es utilizado como aislante de puerta. Pero aislantes demasiado finos conducen a valores de la corriente de fugas inaceptables para un correcto funcionamiento del dispositivo, por lo que la tendencia actual es hacia la búsqueda de aislantes alternativos al $\mathrm{SiO}_{2}\left({ }^{1}\right)$ que tengan una permitividad dieléctrica relativa $\left(\varepsilon_{\mathrm{r}}\right)$ mayor. Esto permitiría espesores físicos mayores, reduciendo así las fugas de corriente a través de la puerta. Es sabido que el nitruro de silicio $\left(\mathrm{Si}_{3} \mathrm{~N}_{4}\right)$ es un dieléctrico alternativo al $\mathrm{SiO}_{2}$ con una $\varepsilon_{\mathrm{r}}$ de $7.4\left(^{2}\right)$, casi el doble que la del $\mathrm{SiO}_{2}$ y con una estructura de enlaces similar. Es imposible hacer crecer $\mathrm{Si}_{3} \mathrm{~N}_{4}$ a partir de los sustratos de $\mathrm{Si}$ a temperaturas aceptables, por lo que el $\mathrm{Si}_{3} \mathrm{~N}_{4}$ ha de ser depositado sobre la superficie del sustrato de Si. Esto puede conducir a la desviación de la composición de las láminas depositadas respecto al valor estequiométrico $\left(\mathrm{SiN}_{1.33}\right)$, así como a la incorporación a la lámina de especies diferentes del silicio y nitrógeno, principalmente hidrógeno. Esto hace que estas láminas se definan como $\mathrm{SiN}_{\mathrm{y}}: \mathrm{H}\left({ }^{3}\right)$. La desviación de la estequiometría, la presencia de hidrógeno y el hecho de que las láminas estén depositadas en lugar de crecidas a partir del sustrato conducen a una intercara $\mathrm{SiN}_{\mathrm{y}}: \mathrm{H} / \mathrm{Si}$ más defectuosa que la del $\mathrm{SiO}_{2} / \mathrm{Si}$, por lo que se hace necesario un procesamiento previo de la superficie del sustrato para minimizar la cantidad de defectos de la intercara aislante/Si.

En este trabajo se propone un proceso de preparación de la superficie del Si consistente en la oxidación de los sustratos mediante un plasma de oxígeno en un reactor de resonancia ciclotrónica de electrones (en adelante ECR). Para ello se han fabricado estructuras MIS sobre Si en el que el dieléctrico de puerta se ha fabricado mediante un 
proceso en dos pasos:

i) Exposición de la superficie de $\mathrm{Si}$ a un plasma ECR de oxígeno para obtener una fina capa de $\mathrm{SiO}_{x}$ (en adelante $\mathrm{PO}-\mathrm{SiO}_{x}$ ) que permita tener una intercara aislante/ sustrato de buena calidad.

ii) Depósito de nitruro de silicio $\left(\mathrm{SiN}_{1.55}: \mathrm{H}\right)$ mediante plasma ECR usando silano y nitrógeno como gases precursores.

Las razones de usar un plasma ECR tanto para oxidar el sustrato como para depositar el $\mathrm{SiN}_{\mathrm{y}}: \mathrm{H}$ son varias. Las principales son las siguientes: los métodos de plasma son capaces de depositar todo tipo de materiales de la familia $\mathrm{Si}-\mathrm{O}-\mathrm{N}$ a bajas temperaturas de proceso, el plasma ECR es un proceso de plasma remoto, por lo que se reduce el posible daño a la superficie generado por bombardeo iónico ${ }^{(4)}$, consiguiéndose un excelente grado de activación de especies $\left(^{5}\right)$, permitiendo el depósito de dieléctricos de puerta a bajas temperaturas $\left(^{6}\right)$; por último, la técnica de plasma ECR permite obtener excelentes características de intercara mediante oxidación por plasma de la superficie del sustrato de $\operatorname{Si}\left({ }^{7}\right)$.

\section{EXPERIMENTAL}

Se depositaron dieléctricos apilados con la estructura $\mathrm{SiN}_{\mathrm{y}}: \mathrm{H} / \mathrm{PO}-\mathrm{SiO}_{\mathrm{x}} / \mathrm{Si}$ sobre dos tipos de sustratos limpiados previamente mediante limpieza RCA: para caracterización capacidad-voltaje (C-V) se usaron sustratos de $\mathrm{Si}$ (100) pulidos por una cara, con $5 \Omega . c m$ de resistividad, tipo $n$ y de $500 \mu \mathrm{m}$ de espesor; para espectroscopia infrarroja por transformada de Fourier (FTIR) se utilizaron obleas de alta resistividad pulidas por ambas caras (80 $\Omega . \mathrm{cm}$, orientación (111)).

Los procesos de oxidación y depósito se realizaron en una cámara de alto vacío fabricada en nuestro laboratorio. El generador del plasma ECR es un reactor modelo Astex 4500. Para más detalles del sistema de depósito ver ref. 6. Los gases precursores fueron $\mathrm{O}_{2}$ de alta pureza para las oxidaciones por plasma, y $\mathrm{SiH}_{4}(1.76 \mathrm{sccm})$ y $\mathrm{N}_{2}(8.76 \mathrm{sccm})$ para el depósito de $\mathrm{SiN}_{\mathrm{y}}: \mathrm{H}$. Esta mezcla de gases produce láminas con un valor de $y$ de $1.55\left(^{(}\right)$. Para caracterizar el proceso de oxidación por plasma se variaron los siguientes parámetros: el tiempo de depósito del $\mathrm{SiN}_{1.55}: \mathrm{H}$ entre 4 y $13 \mathrm{~min}$, el tiempo de oxidación entre 10 y 180 min, la temperatura del sustrato entre 150 y $290^{\circ} \mathrm{C}$ y el flujo de $\mathrm{O}_{2}$ entre 10.5 y $60 \mathrm{sccm}$. La potencia de microondas se mantuvo fija en $100 \mathrm{~W}$ en todos los casos.

Una vez fabricados los dieléctricos apilados sobre Si de $5 \Omega . c m$, se depositaron cuadrados de $\mathrm{Al}$ con una superficie media de 0.124 $\mathrm{mm}^{2}$ mediante evaporación por haz de e. También se depositó Al en la parte trasera de las muestras para formar el electrodo trasero. En los dispositivos MIS así fabricados se midieron las características C-V a alta frecuencia $\left(C_{h^{\prime}} 1 \mathrm{MHz}\right)$ y cuasiestática $\left(C_{q}\right)$ simultáneamente. El sistema de medida utilizado es un Keithley 82. De ambas características se obtuvo la distribución de trampas en la intercara $\left(D_{i t}\right)\left({ }^{9}\right)$.

Las muestras depositadas sobre sustratos de alta resistividad se caracterizaron mediante FTIR en el rango $350-4000 \mathrm{~cm}^{-1}$ con un espectrómetro modelo Nicolet Magna-IR 750 series II trabajando en el modo de transmisión.

\section{RESULTADOS Y DISCUSIÓN}

El primer objetivo fue garantizar que el proceso de oxidación por plasma permite el crecimiento de películas de $\mathrm{PO}-\mathrm{SiO}_{x}$ de espesor adecuado. Para comprobarlo, se utilizó el método propuesto en la referencia $\left({ }^{10}\right)$, en el que a partir de la capacidad en acumulación del dieléctrico apilado se calculan los espesores de cada una de las láminas: si se supone que el dieléctrico apilado forma una estructura de condensadores en serie se ha de cumplir que:

$$
\frac{1}{C_{a c}}=\frac{v_{\text {crec }}}{A_{\text {elec }} \mathcal{E}_{o} \mathcal{E}_{S N_{1.55}: H}} t_{d e p}+\frac{d_{P O-S i O_{x}}}{A_{\text {elec }} \mathcal{E}_{o} \mathcal{E}_{P O-S i O_{x}}}
$$

siendo $C_{a c}$ la capacidad total del dispositivo MIS en acumulación, $t_{\text {dep }}$ el tiempo de depósito del $\mathrm{SiN}_{1,55}: \mathrm{H}$ y $v_{\text {crec }}$ su velocidad de depósito, $A_{\text {elec }}$ el área del electrodo de $\mathrm{Al}, \varepsilon_{o}$ la permitividad dieléctrica del vacío, $\mathcal{E}_{S i_{1.55}: H}$ y $\mathcal{E}_{\text {PO-SiO }_{x}}$ las permitividades relativas del $\mathrm{SiN}_{1.55}: \mathrm{H}$ y $\mathrm{PO}-\mathrm{SiO}_{x}$ respectivamente, y $d_{P O-S i O_{x}}$ el espesor del óxido. Se preparó una serie de muestras oxidadas por plasma ECR durante $180 \mathrm{~min}$ en las que el $\mathrm{SiN}_{1.55}: \mathrm{H}$ se depositó durante diferentes tiempos (desde 4 hasta $13 \mathrm{~min}$ ). Se supusieron unos valores de $6.35\left({ }^{11}\right)$ y $3.8(1)$ y para $\mathcal{E}_{\mathcal{S i N}_{1.55}: H}$ y $\mathcal{E}_{P O-S i O_{x}}$ respectivamente. A partir del ajuste a una recta de la inversa de la capacidad total en acumulación frente al tiempo de depósito del $\mathrm{SiN}_{1.5}: \mathrm{H}$ se pudo obtener la velocidad de crecimiento del $\mathrm{SiN}_{1.55}: \mathrm{H}$ y el espesor de la capa de $\mathrm{PO}-\mathrm{SiO}_{x}$. Dicho ajuste se presenta en la figura 1, donde se presentan los valores de la inversa de la capacidad en acumulación para diversos dispositivos MIS con dieléctrico apilado. En dichos dispositivos el $\mathrm{SiN}_{1.55}: \mathrm{H}$ se depositó para tres tiempos diferentes (4, 9 y 13 min). El ajuste por mínimos cuadrados de los datos experimentales a la ecuación [1] conduce a unos valores para $v_{\text {crec }}$ de $5.46 \mathrm{~nm} \cdot \mathrm{min}^{-1} \mathrm{y}$ para $d_{P O-S O_{\mathrm{X}}}$ de $10.4 \mathrm{~nm}$. Para corroborar que el método para calcular dicho espesor conduce a resultados correctos, en la figura 1 se han incluido también los valores de la inversa de la capacidad en acumulación de varios dispositivos MIS oxidados por plasma ECR a $200^{\circ} \mathrm{C}$ durante $180 \mathrm{~min}$ solamente $\left(\mathrm{PO}-\mathrm{SiO}_{x} / \mathrm{Si}\right)$. Se comprueba que dichos valores concuerdan perfectamente con el ajuste obtenido con los dieléctricos apilados. Suponiendo que dicho dispositivo es un condensador simple, a partir de la media de los valores de la capacidad en acumulación de los dispositivos sin capa de $\mathrm{SiN}_{1.55}: \mathrm{H}$ se pudo obtener otra estimación del espesor de la capa de $\mathrm{PO}-\mathrm{SiO}_{\mathrm{x}}$. El valor que se obtuvo en ese caso es de $11.0 \mathrm{~nm}$, muy similar al valor encontrado previamente, lo que confirma la validez del método.

Una vez comprobado mediante las medidas eléctricas que el proceso de oxidación por plasma ECR permite obtener $\mathrm{PO}_{-} \mathrm{SiO}_{x}$ se pasó a caracterizar dicho proceso de oxidación. Para ello se fabricaron sobre $\mathrm{Si}$ de alta resistividad estructuras de dieléctrico apilado $\mathrm{SiN}_{1.55}: \mathrm{H} / \mathrm{PO}-\mathrm{SiO}_{x}$ variando en este caso el tiempo de oxidación por

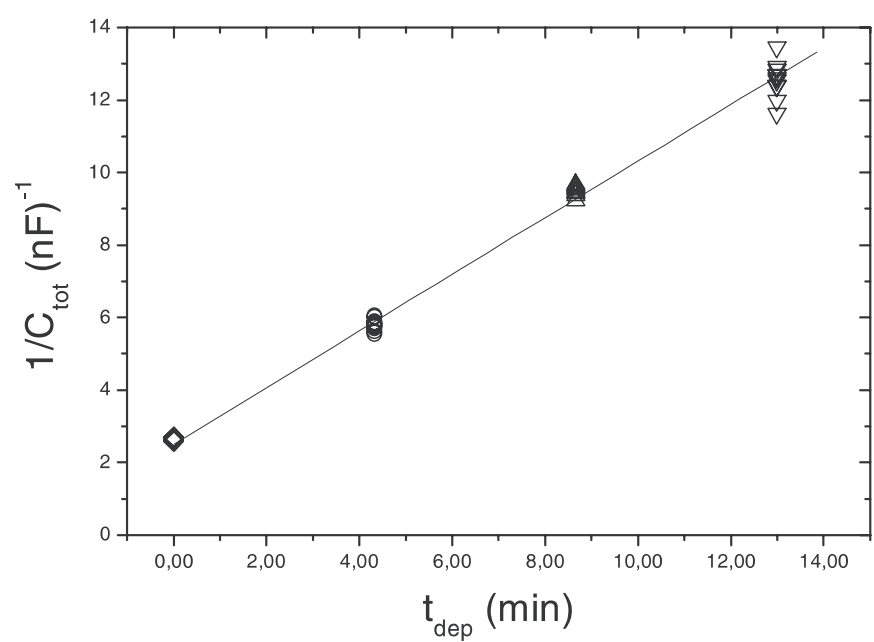

Figura 1: Inversa de la capacidad en acumulación de varios dispositivos con dieléctrico de puerta de la forma $\mathrm{SiN}_{155}: \mathrm{H} / \mathrm{PO}-\mathrm{SiO}_{x}$ oxidados simultáneamente durante $180 \mathrm{~min}$ a $200^{\circ} \mathrm{C}$ en los que se ha variado el tiempo de depósito del $\operatorname{SiN}_{155}: \mathrm{H}\left(O 4^{\prime}, \triangle 9^{\prime}, \nabla 13^{\prime}\right)$, y un dispositivo sin depósito de $\mathrm{SiN}_{1.55}: \mathrm{H}$, solo con $\mathrm{PO}^{-S_{\mathrm{O}}}{ }_{\mathrm{x}}\left(\diamond, \mathrm{t}_{\text {dep }}=0\right)$ 
plasma. En la figura 2 se muestran los espectros de FTIR de cinco dieléctricos apilados en los que el proceso de oxidación tuvo una duración entre 10 y $180 \mathrm{~min}$. La lámina de $\mathrm{SiN}_{1.5}: \mathrm{H}$, depositada simultáneamente en todos los casos, tuvo un espesor de $25 \mathrm{~nm}$. Como referencia se incluye el espectro de una lámina de $\mathrm{SiN}_{\mathrm{x}}: \mathrm{H}$ sin el proceso de oxidación por plasma. Aunque el espectro ha sido medido en la región espectral entre $350-4000 \mathrm{~cm}^{-1}$, los modos espectrales más interesantes se localizan en la región entre $700-1350 \mathrm{~cm}^{-1}$, así que ésta es la región que se presenta en la figura 2 .

Cada espectro muestra claramente dos picos principales:

i) Un pico más intenso, idéntico para todas las láminas, con su máximo en $852 \mathrm{~cm}^{-1}$ y una anchura a mitad del máximo (FWHM) de $171 \mathrm{~cm}^{-1}$. Este pico es el conocido modo stretching del enlace Si-N ${ }^{(2)}$, y su forma es idéntica en todas los espectros. Esto es debido a que el $\mathrm{SiN}_{1.55}: \mathrm{H}$ se depositó simultáneamente en todas las muestras.

ii) Otro pico menos intenso, con su máximo localizado en 1059 $\mathrm{cm}^{-1} \mathrm{y}$ una FWHM de $77 \mathrm{~cm}^{-1}$. Este pico es la bien conocida vibración stretching del enlace Si-O-Si $\left({ }^{13}\right)$ en un entorno rico en $\mathrm{Si}$. La composición de la lámina de $\mathrm{PO}-\mathrm{SiO}_{\mathrm{x}}$ puede ser estimada a partir de la posición del máximo de dicho modo ${ }^{\left({ }^{14}\right)}$. La composición que se obtiene es $\mathrm{SiO}_{1.8}$. Para analizar el modo Si-O-Si stretching se sustrae a cada uno de los espectros de los dieléctricos apilados el espectro de la muestra sin el proceso de oxidación por plasma. Los resultados de dichas sustracciones se muestran en el detalle de la figura 2, que muestra cómo según aumenta el tiempo de exposición al plasma el pico debido al enlace Si-O-Si aumenta de área. Es conocido que el $\mathrm{SiO}_{2}$ térmico no crece linealmente con el tiempo $\left({ }^{15}\right)$. Lo mismo ocurre con el $\mathrm{PO}-\mathrm{SiO}_{x^{\prime}}$ como se muestra en la figura 3 , en la que se muestra el área del pico Si-O-Si frente al tiempo de oxidación. Como dicha área está en relación directa con el espesor de la lámina, se puede obtener la ley que rige el crecimiento de la lámina frente al tiempo de oxidación por plasma ECR. El ajuste de los datos de la fig. 3 conduce a una ley de la forma $\mathrm{A}_{\mathrm{SiO}}=0.25 \mathrm{t}_{\mathrm{ox}}{ }^{0.26}$. Dicho ajuste se presenta también en la figura 3 con una línea continua. Para obtener el parámetro pre-exponencial de la ley de crecimiento se utiliza un resultado anterior: los resultados eléctricos mostraron que el espesor de la lámina oxidada durante $180 \mathrm{~min}$ es de $10.4 \mathrm{~nm}$, luego introduciendo dicho valor en la fórmula anterior se obtiene el parámetro pre-exponencial. De este modo, la ley resulta ser finalmente $d_{\text {po-siox }}=2.7 t_{\text {ox }}{ }^{0.26}$, donde el tiempo de oxidación se expresa en min y el espesor en $\mathrm{nm}$. Lucovsky et al. encontró que esta ley era $\mathrm{d}_{\mathrm{ox}}=0.7 \mathrm{t}_{\mathrm{ox}}{ }^{0.28}$ cuando oxidaban $\mathrm{Si}$ (100) en un sistema de plasma remoto CVD $\left({ }^{16}\right)$. La coincidencia en el parámetro exponencial de ambas leyes es excepcional, pero el factor de proporcionalidad en nuestro caso es casi 4 veces mayor. Esta diferencia es fácilmente explicada por el mayor grado de activación de las especies que el plasma ECR es capaz de conseguir comparado con otros métodos de plasma CVD (17), menos eficientes en la activación de especies.

Asimismo se estudió la influencia del flujo total de $\mathrm{O}_{2}$ y de la temperatura del sustrato durante el proceso de oxidación en la estructura de enlaces del $\mathrm{PO}_{-} \mathrm{SiO}_{x}$ y en las características eléctricas de los dispositivos MIS. Para ello se realizaron dos series de muestras: una con $30 \mathrm{sccm}$ de flujo de $\mathrm{O}_{2}$ variando la temperatura de sustrato entre $150^{\circ} \mathrm{C}$ y $290^{\circ} \mathrm{C}$, y otra a $200^{\circ} \mathrm{C}$ variando el flujo de $\mathrm{O}_{2}$ entre $10,5 \mathrm{sccm}$ y $60 \mathrm{sccm}$.

A partir de la caracterización infrarroja concluimos que la influencia de ambos parámetros no es significativa en la posición ni en el área del pico stretching del enlace Si-O-Si. Sin embargo, en las láminas depositadas a $200^{\circ} \mathrm{C}$ con $30 \mathrm{sccm}$ de flujo de $\mathrm{O}_{2}$ el FWHM del pico Si-O-Si es $76 \mathrm{~cm}^{-1}$, valor ligeramente inferior al resto de casos $\left(80-84 \mathrm{~cm}^{-1}\right)$. Esto es una indicación de una mayor calidad $\left({ }^{18}\right)$ de las láminas de $\mathrm{PO}-\mathrm{SiO}_{x}$ depositadas en estas condiciones, ya que este parámetro es una indicación del desorden estructural de la lámina ${ }^{\left({ }^{19}\right)}$.

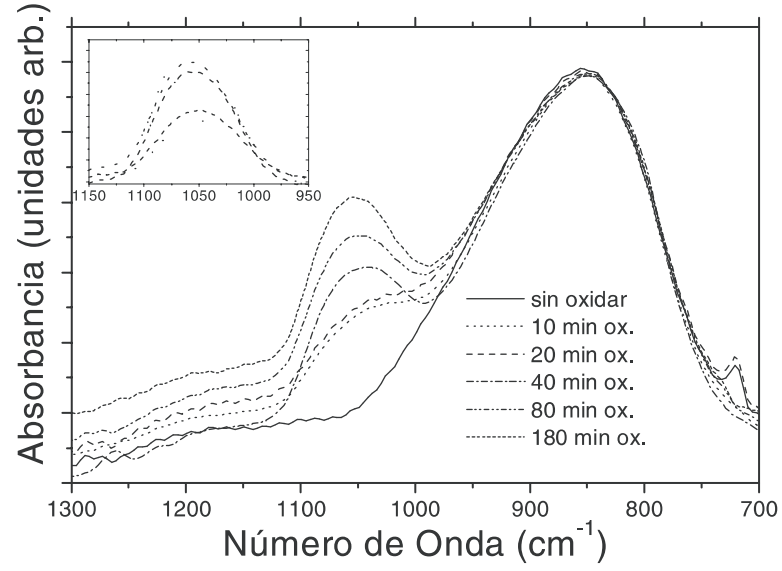

Figura 2: Espectro FTIR de una lámina de $\mathrm{SiN}_{1.55}: \mathrm{H}$ (?), y de cinco dieléctricos apilados $\mathrm{SiN}_{155}: \mathrm{H} / \mathrm{PO}-\mathrm{SiO}_{\mathrm{x}^{\prime}}$ oxidados durante diferentes tiempos: $10 \mathrm{~min}(\bullet \bullet \bullet \bullet), 20 \mathrm{~min}(-----), 40 \mathrm{~min}(-\bullet-\bullet-\bullet-\bullet-), 80 \mathrm{~min}$

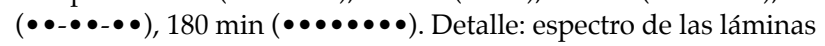
oxidadas después de la sustracción del espectro del $\mathrm{SiN}_{1.55}: \mathrm{H}$.

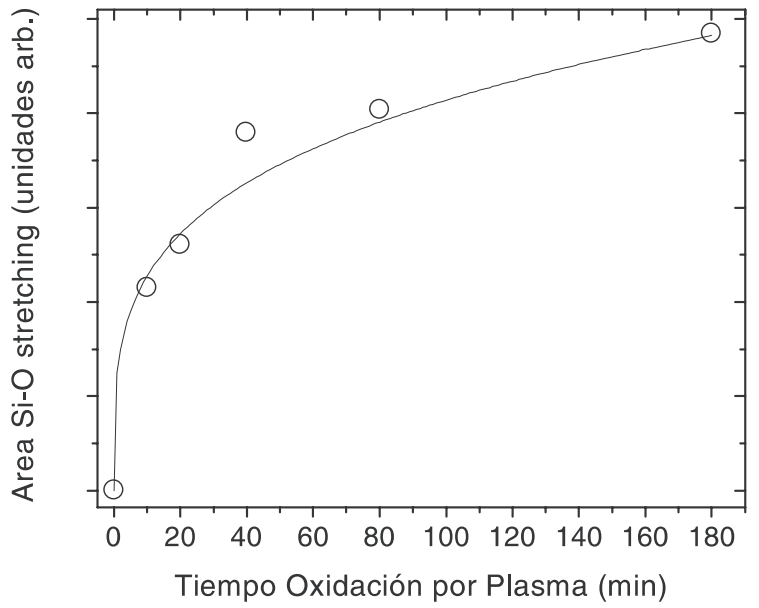

Figura 3: Área del pico Si-O-Si stretching frente al tiempo de oxidación por plasma (o) y ajuste de dichos datos a la ley $\mathrm{A}_{\mathrm{SiO}}=0.25 \mathrm{t}_{\mathrm{ox}}^{0.26}$

La caracterización eléctrica de los dispositivos MIS muestra que todos los dispositivos tienen unas características eléctricas similares, con mínimos en la densidad de trampas en la intercara en torno a $10^{11} \mathrm{eV}^{-1} \mathrm{~cm}^{-2}$. En la figura 4 se muestran las características $\mathrm{C}-\mathrm{V}$ de una muestra oxidada a $200^{\circ} \mathrm{C}$ durante $60 \mathrm{~min}$ con un flujo de $\mathrm{O}_{2}$ de 30 sccm, seguida de un depósito de $50 \mathrm{~nm}$ de $\mathrm{SiN}_{1.55}: \mathrm{H}$. En la figura $5 \mathrm{se}$ muestra la concentración de trampas en la intercara para el dispositivo de la figura 4. Para mostrar cómo el proceso de oxidación por plasma mejora las características de los dispositivos fabricados solamente con $\mathrm{SiN}_{1.55}: \mathrm{H}$, se incluye en la figura 5 la concentración de trampas en la intercara de un dispositivo procesado simultáneamente con el dispositivo de la figura 4 salvo el proceso de oxidación ${ }^{(20)}$. En dicha figura se comprueba cómo el dispositivo con la lámina de $\mathrm{PO}_{-} \mathrm{SiO}_{x}$ mejora las características del dispositivo fabricado solamente con $\mathrm{SiN}_{1.55}: \mathrm{H}$, tanto en el mínimo de la densidad de trampas $\left(1.2 \times 10^{11} \mathrm{eV}^{-1} \mathrm{~cm}^{-2}\right.$ frente a $\left.6 \times 10^{11} \mathrm{eV}^{-1} \mathrm{~cm}^{-2}\right)$ como en el barrido del nivel de Fermi $(0.93 \mathrm{eV}$ frente a $0.78 \mathrm{eV})$. Medidas consecutivas de la capacidad acumulación-inversión e inversión-acumulación en alta frecuencia muestran asimismo que también es menor la histéresis eléctrica de los dispositivos con capa de $\mathrm{PO}-\mathrm{SiO}_{\mathrm{x}}(100 \mathrm{mV}$ frente a $150 \mathrm{mV})$. A pesar de que en todos los casos las características eléctricas son similares, los dispositivos en los que el $\mathrm{PO}-\mathrm{SiO}_{x}$ se ha crecido a $200^{\circ} \mathrm{C}$ con $30 \mathrm{sccm}$ de flujo de $\mathrm{O}_{2}$ presentaron una mayor reproducibilidad que el resto de casos. 


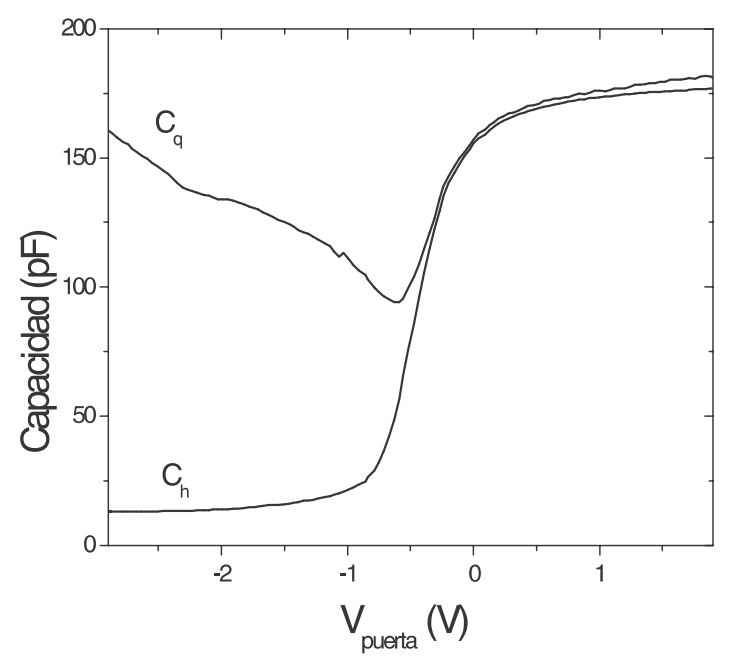

Figura 4: Capacidades de alta frecuencia y cuasiestática de un dispositivo fabricado con una estructura $\mathrm{SiN}_{1.55}: \mathrm{H} / \mathrm{PO}-\mathrm{SiO}_{x}$ como dieléctrico de puerta. La oxidación de la superficie se realizó durante $180 \mathrm{~min}$ a $200^{\circ} \mathrm{C}$ y con un flujo de $\mathrm{O}_{2}$ de $30 \mathrm{sccm}$. $\mathrm{El} \mathrm{SiN}_{1.55}: \mathrm{H}$ tiene un espesor de $50 \mathrm{~nm}$.

\section{CONCLUSIONES}

Mediante un plasma ECR de $\mathrm{O}_{2}$ se ha conseguido hacer crecer una lámina de subóxido de $\mathrm{Si}$ a partir de sustratos de $\mathrm{Si}$, tal y como se ha comprobado mediante medidas eléctricas. El análisis de FTIR muestra que dicho subóxido tiene una composición igual a $\mathrm{SiO}_{18}$ y que la ley que rige el espesor del $\mathrm{PO}-\mathrm{SiO}_{\mathrm{x}}$ crecido frente al tiempo es $\mathrm{d}_{\mathrm{siO}}=2.7$ $t_{o x}{ }^{0.26}$. Dicha ley coincide en forma y valores con resultados publicados por otros grupos, salvo el término pre-exponencial, que es superior en nuestro caso. Esta discrepancia se explica por la mayor activación de especies que consiguen los plasmas ECR frente a otros métodos de generar la descarga. La variación del flujo total de $\mathrm{O}_{2}$ así como la variación de la temperatura del sustrato tienen una débil influencia en las características de las láminas de $\mathrm{PO}-\mathrm{SiO}_{x}$ crecidas. La introducción de la lámina de $\mathrm{PO}_{-} \mathrm{SiO}_{x}$ en dispositivos MIS fabricados con $\mathrm{SiN}_{1.55}: \mathrm{H}$ como aislante de puerta hace que las características eléctricas de dichos dispositivos mejoren, presentando mínimos de trampas en la intercara en torno a $10^{11} \mathrm{eV}^{-1} \mathrm{~cm}^{-2}$.

\section{AGRADECIMIENTOS}

Los autores agradecen al C.A.I. de Implantación Iónica (U.C.M.) por las labores técnicas de apoyo, y al C.A.I. de Espectroscopía (U.C.M.) por la disponibilidad del espectrómetro FTIR. Éste trabajo fue parcialmente financiado por el CICYT bajo contrato TIC 01-1253.

\section{BIBLIOGRAFÍA}

1. G. D. Wilk, R. M. Wallace, J. M. Anthony, “High-k gate dielectrics: Current status and materials properties considerations", J. Appl. Phys. 89, 5243-5275 (2001).

2. S.M. Sze, "Physics of Semiconductor Devices", John Willey \& Sons (1981).

3. F. L. Martínez, I. Mártil, G. González-Díaz, B. Selle, I. Sieber, “Influence of rapid thermal annealing processes on the properties of $\mathrm{SiN}_{\mathrm{x}}: \mathrm{H}$ films deposited by the ECR method", J. Non-Cryst. Sol. 227-230, 523-527 (1998).

4. M. Losurdo, P. Capezzuto, G. Bruno, G. Perna, V. Capozzi, “ $\mathrm{N}_{2}-\mathrm{H}_{2}$ remote plasma nitridation for GaAs surface passivation" App. Phys. Lett. 81, 16-18 (2002).

5. P. K. Shufflebotham, D. J. Thomson, H. C. Card, “Behavior of downstream plasmas generated in a microwave plasma chemical-vapor deposition reactor" J. Appl. Phys. 64, 4398-4403 (1988)

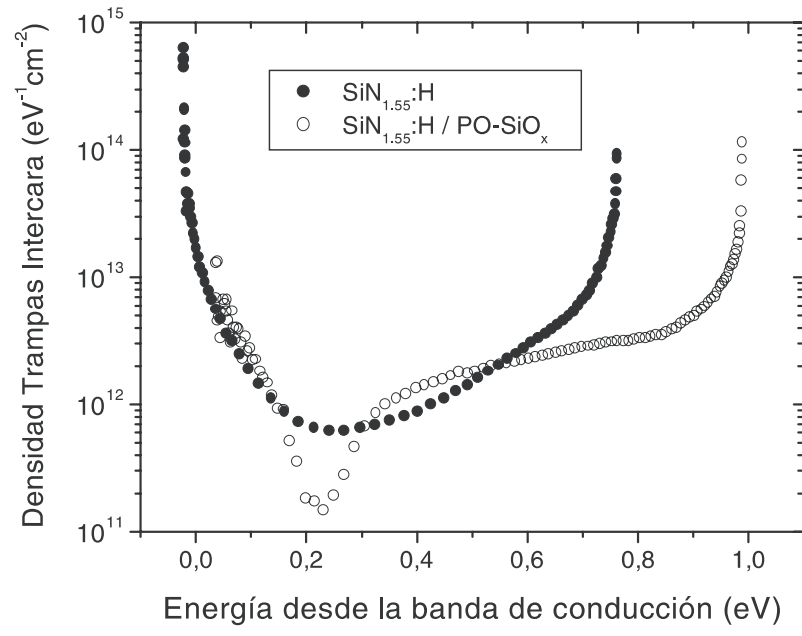

Figura 5: Densidad de trampas en la intercara referidas al fondo de la banda de conducción obtenidas de las curvas $\mathrm{C}-\mathrm{V}$ del dispositivo de la figura 4 (o) y de un dispositivo sin oxidar, sólo con $\mathrm{SiN}_{155}: \mathrm{H}$ como dieléctrico de puerta $(\bullet$

6. F. L. Martínez, E. San Andrés, A. del Prado, I. Mártil, D. Bravo, F. J. López, “Temperature effects on the elecrical properties and structure of interfacial and bulk defects in $\mathrm{Al} / \mathrm{SiN}: \mathrm{H} / \mathrm{Si}$ devices" J. Appl. Phys. 90, 1573-1581 (2001).

7. K. Miyake, S. Kimura, T. Warabisako, H. Sunami, and T. Tokuyama, "Microwave plasma stream transport system for low temperature plasma oxidation" J. Vac. Sci. Technol. A 2, 496-499 (1984).

8. E. San Andrés, A. Del Prado, F. L. Martinez, I. Mártil, D. Bravo and F. J. López, “Rapid thermal annealing effects on the structural properties and density of defects in $\mathrm{SiO}_{2}$ and $\mathrm{SiN}_{\mathrm{x}}: \mathrm{H}$ films deposited by electron cyclotron resonance", J. Appl. Phys. 87, 1187-1192 (2000)

9. E. H. Nicollian and J. R. Brews, MOS (Metal-Oxide-Semiconductor) Physics and Technology (Willey, New York, 1982).

10. M. Houssa, R. Degraeve, P. W. Mertens, M. M. Heyns, J. S. Jeon, A. Halliyal, B. Ogle, "Electrical properties of thin $\mathrm{SiON} / \mathrm{Ta}_{2} \mathrm{O}_{5}$ gate dielectric stacks" J. Appl. Phys. 86, 6462-6467 (1999)

11. F. L. Martínez, E. San Andrés, A. del Prado, I. Mártil, D. Bravo, F. J. López, "temperatura effects on the electrical properties and structure of interfacial and bula defects in $\mathrm{Al} / \mathrm{SiN}_{\mathrm{x}}: \mathrm{H} / \mathrm{Si}$ devices" J. Appl. Phys. 90, 1573-1581 (2001).

12. W. R. Knolle, J. W. Osenbach, "The structure of plasma-deposited silicon nitride films determined by infrared spectroscopy" J. Appl. Phys. 58, 1248-1254 (1985)

13. J. Lubguban Jr., Y. Kurata, T. Inokuma, S. Hasegawa, "Thermal stability and breakdown strenght of carbon-doped $\mathrm{SiO}_{2}: \mathrm{H}$ films prepared by plasma-enhanced chemical vapor deposition method" J. Appl. Phys. 87, 3715-3722 (2000)

14. D. V. Tsu, G. Lucovsky and B. N. Davidson. "Effects of the nearest neighbours and the alloy matrix on $\mathrm{SiH}$ stretching vibrations in the amorphous $\mathrm{SiO}_{\mathrm{r}}: \mathrm{H}(0<\mathrm{r}<2)$ alloy system" Phys. Rev. B 40, 1795-1805 (1989).

15. Wolf and Tauber "Silicon Processing for the VLSI Era, Vol.1: Process Technology", Lattice Press, 1986.

16. G. Lucovsky, "Ultrathin nitrided gate dielectrics: Plasma processing, chemical characterization, performance, and reliability",IBM J. Res. Develop. 43, 301-326 (1999).

17. A. Popov, "Characteristics of electron cyclotron resonance plasma sources”, J. Vac. Sci. Technol. A 7, 894-898 (1989).

18. E. San Andrés, A. del Prado, I. Mártil, G. González-Díaz, D. Bravo, F. J. López, "Thermally induced modifications on bonding configuration and density of de-

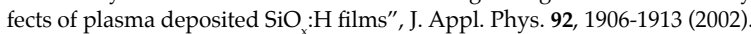

19. K. Furukawa, Y. Liu, H. Nakashima, D. Gao, K. Uchino, K. Muraoka and H. Tsuzuki. "Observation of $\mathrm{Si}$ cluster formation in $\mathrm{SiO}_{2}$ films through annealing process using x-ray photoelectron spectroscopy and infrared techniques" Appl. Phys. Lett. 72, 725-727 (1998).

20. F. L. Martínez, A. del Prado, I. Mártil, G. González-Díaz, W. Bohne, W. Fuhs, J. Röhrich, B. Selle, I. Sieber, "Molecular models and activation energies for bonding rearrangement in plasma-deposited a-SiN $\mathrm{x}: \mathrm{H}$ dielectric thin films treated by rapid thermal annealing" Phys. Rev B 63, 245320 1-11(2001)

Recibido: 1.2 .03

Aceptado: 30.11 .03 